Journal of Computer Science 4 (4): 305-308, 2008

ISSN 1549-3636

(C) 2008 Science Publications

\title{
Performance Analysis of a 32-Bit Multiplier with a Carry-Look-Ahead Adder and a 32-bit Multiplier with a Ripple Adder using VHDL
}

\author{
Hasan Krad and Aws Yousif Al-Taie \\ Department of Computer Science and Engineering, \\ College of Engineering, Qatar University, P.O. Box 2713, Doha, Qatar
}

\begin{abstract}
This study presents a performance analysis of two different multipliers for unsigned data, one uses a carry-look-ahead adder and the second one uses a ripple adder. The study's main focus is on the speed of the multiplication operation on these 32-bit multipliers which are modeled using VHDL, A hardware description language. The multiplier with a carry-look-ahead adder has shown a better performance over the multiplier with a ripple adder in terms of gate delays. Under the worst case, the multiplier with the fast adder shows approximately twice the speed of the multiplier with the ripple adder. The multiplier with a ripple adder uses time $=979.056 \mathrm{~ns}$, while the multiplier with the carrylook-ahead adder uses time $=659.292 \mathrm{~ns}$.
\end{abstract}

Key words: Multiplier, carry-look-ahead adder, ripple adder, VHDL simulation

\section{INTRODUCTION}

Multiplication can be considered one of the basic arithmetic operations. However, it is not as simple as addition or subtraction operations, because it takes more time to perform two subtasks, addition and shifting. Typically, a multiplication operation takes between 2 and 8 cycles $^{[2]}$. Therefore, using high-speed multipliers is a critical requirement for processors with a high performance. The multiplier uses the addition operation for all the partial products. The adder can be a ripple adder, a carry-look-ahead adder, or any other adder ${ }^{[5,8]}$. However, using a fast adder for the multiplier improves the over all performance of the multiplication operation. Our study is focused on multipliers using unsigned data. VHDL, a Very High Speed Integrated Circuit Hardware Description Language, was used to model our multiplier design.

Several researchers had worked on the performance analysis of adders and other researchers on the performance analysis of multipliers. Sertbas and Selami worked on the performance analysis of classified binary adder architectures. They compared the ripple adder, carry-look-ahead adder, carry select adder and the conditional sum adder. They used VHDL in their comparison. Their study included the unit-gate models for area and delay ${ }^{[1]}$. Asadi and Navi developed a new $54 \times 54$ bit multiplier using a high-speed carrylook-ahead adder. Their proposed multiplier reduced the number of transistors, delay and power consumption $^{[2]}$. Aziz and Kamruzzaman developed a synthesizable VHDL model for a generalized signed multiplier capable of performing multiplication of both signed-magnitude and two's compliment operands ${ }^{[3]}$.

Ripple carry adder: Ripple carry adders use multiple full adders with the carry ins and carry outs chained together, where the correct value of the carry bit ripples from one bit to the next ${ }^{[4]}$.

The two Boolean functions for the sum and carry are:

$$
\begin{aligned}
& \mathrm{SUM}=\mathrm{A}_{\mathrm{i}} \oplus \mathrm{B}_{\mathrm{i}} \oplus \mathrm{C}_{\mathrm{i}} \\
& \mathrm{C}_{\text {out }}=\mathrm{C}_{\mathrm{i}+1}=\mathrm{A}_{\mathrm{i}} \cdot \mathrm{B}_{\mathrm{i}}+\left(\mathrm{A}_{\mathrm{i}} \oplus \mathrm{B}_{\mathrm{i}}\right) \cdot \mathrm{C}_{\mathrm{i}}
\end{aligned}
$$

We modeled this module with the following VHDL code:

ENTITY R_Adder IS

PORT (

a,b: IN BIT_VECTOR (31 DOWNTO 0); cin: IN BIT; sum: OUT BIT_VECTOR (31 DOWNTO 0); cout: OUT BIT

) ;

END Entity;

ARCHITECTURE R_Adder_Beh OF R_Adder IS constant gate_delay2: time: $=10 \mathrm{~ns}$; constant gate_delay4: time: $=20 \mathrm{~ns}$;

Corresponding Author: Hasan Krad, Department of Computer Science and Engineering, College of Engineering, Qatar University, P.O. Box 2713, Doha, Qatar Tel: +974 485-2677 Fax: +974 485-2777 
Multiplicand $=32$ bits

Register $=64$ bits

Put the multiplier in the least significant half and clear the most significant half

For $\mathrm{i}=1$ to 32

Begin Loop

If the least significant bit of the 64-bit register contains binary ' 1 '

Begin If

Add the Multiplicand to the Most Significant

Half using the CLAA

Begin Adder

$\mathrm{C}[0]={ }^{\prime} 0$ '

For $\mathrm{j}=0$ to 31

Begin Loop

Calculate Propagate $\mathrm{P}[\mathrm{j}]=$ Multiplicand $[\mathrm{j}]$

$\oplus$ Most Significant Half[j]

Calculate Generate $\mathrm{G}[\mathrm{j}]=$

Multiplicand[j].Most Significant Half[j]

Calculate Carries $\mathrm{C}[\mathrm{i}+1]=\mathrm{G}[\mathrm{i}]+\mathrm{P}[\mathrm{i}]$.

$\mathrm{C}[\mathrm{i}]$

$$
\text { Calculate Sum S[i] = P }[\mathrm{i}] \oplus \mathrm{C}[\mathrm{i}]
$$

End Loop

End Adder

Shift the 64-bit Register one bit to the right throwing away the least significant bit

Else

Only Shift the 64-bit Register one bit to the right throwing away the least significant bit End If

End Loop

Register $=$ Sum of Partial Products

End Program

\section{Algorithm for a Multiplier with a Ripple Adder:}

Begin Program

Multiplier $=32$ bits

Multiplicand $=32$ bits

Register $=64$ bits

Put the multiplier in the least significant half and clear the most significant half

For $\mathrm{i}=1$ to 32

Begin Loop

If the least significant bit of the 64-bit register contains binary ' 1 '

Begin If

Add the Multiplicand to the Most Significant

Half using the RA

Begin Adder

$\mathrm{C}[0]={ }^{\prime} 0$ '

For $\mathrm{j}=0$ to 31

Begin Loop
Calculate Sum S[j] = Multiplicand $[\mathrm{j}] \oplus$ Most Significant Half $[\mathrm{j}] \oplus \mathrm{C}[\mathrm{j}]$

Calculate Carries $\mathrm{C}[\mathrm{j}+1]=$ Multiplicand $[\mathrm{j}]$. Most Significant Half[j] + (Multiplicand[j.] $\oplus$ Most Significant Half[j]) $\cdot \mathrm{C}[\mathrm{j}]$

End Loop

End Adder

Shift the 64-bit Register one bit to the right throwing away the least significant bit

Else

Only Shift the 64-bit Register one bit to the right throwing away the least significant bit

\section{End If}

End Loop

Register $=$ Sum of Partial Products

End Program

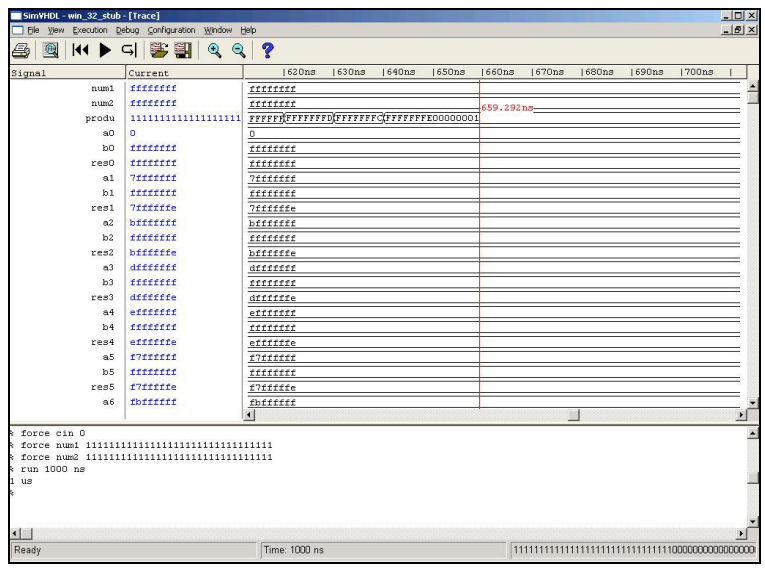

Fig. 1: Waveform and command line window for the 32-bit multiplier using a carry look ahead adder

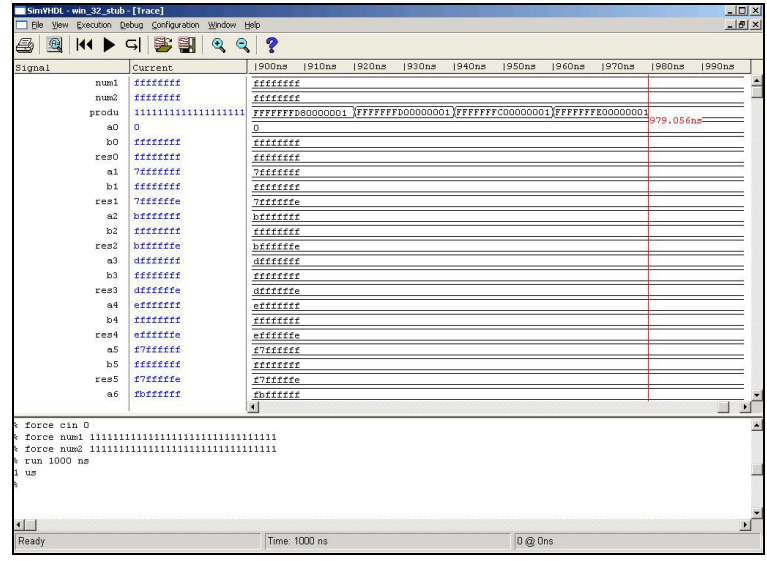

Fig. 2: Waveform and command line window for the 32-bit multiplier using a ripple adder 
Future work: This study can be extended to cover signed multipliers using VHDL.

\section{CONCLUSION}

Two different multipliers using a fast carry-lookahead adder and a ripple adder have been modeled and simulated using VHDL. The multiplier with a carrylook-ahead adder has shown a better performance over the multiplier with a ripple adder in terms of gate delays. In other words, the multiplier with the carrylook-ahead adder has approximately twice the speed of the multiplier with the ripple adder, under the worst case. In fact, the multiplier with the carry-look-ahead adder uses time $=659.292 \mathrm{~ns}$, Fig. $1^{[9]}$, while the multiplier with a ripple adder uses time $=979.056 \mathrm{~ns}$, Fig. $2^{[9]}$.

\section{ACKNOWLEDGEMENT}

We are very grateful to Dr. Belaid Moa for his valuable comments on this study.

\section{REFERENCES}

1. Sertbas, A. and R.S. Özbey, 2004. A performance analysis of classified binary adder architectures and the VHDL simulations. J. Elect. Electron. Eng., Istanbul, $\quad$ Turkey, 4: 1025-1030. http://www.istanbul.edu.tr/eng/ee/jeee/main/pages/i ssues/is41/41005.pdf.

2. Asadi, P. and K. Navi, 2007. A novel high-speed 54-54 bit multiplier. Am. J. Applied Sci., 4 (9): 666-672.

http://www.scipub.org/fulltext/ajas/ajas49666672.pdf.
3. Aziz, S.M., C.N. Basheer and J. Kamruzzaman, 2002. A synthesizable VHDL model for an easily testable generalized multiplier. In: Proceeding of the 1st IEEE International Workshop on Electronic Design, Test Application (DELTA.02), Jan. 29-31, 2002, IEEE Computer Society, Washington, DC, USA, $\quad$ pp: 504-506. http://portal.acm.org/citation.cfm?id=789090.7899 $67 \&$ coll=GUIDE\&dl=GUIDE $\&$ CFID $=40436358 \&$ $\underline{\text { CFTOKEN }=40390034}$

4. Stephen Brown and Zvonko Vranesic, 2005. Fundamentals of Digital Logic with VHDL Design. 2nd Edn. McGraw-Hill Higher Education, USA. ISBN: 0072499389.

5. William Stallings, 2006. Computer Organization and Architecture Designing for Performance. 7th Edn. Pearson Prentice Hall, USA. ISBN: 0-13185644-8.

6. Armstrong, J.R. and F.G. Gray, 2000. VHDL Design Representation and Synthesis. 2nd Edn. Prentice Hall, USA. ISBN: 0-13-021670-4.

7. Zainalabedin Navabi, 2007. VHDL Modular Design and Synthesis of Cores and Systems. 3rd Edn. McGraw-Hill Professional, USA. ISBN: 9780071508926.

8. Wakerly, J.F., 2006. Digital Design-Principles and Practices. 4th Edn. Pearson Prentice Hall, USA. ISBN: 0131733494.

9. Software Simulation Package: DrirectVHDL, Version 1.2, 2007, Green Mounting Computing Systems, Inc., Essex, VT, USA. http://www.gmvhdl.com 